\title{
In vivo polarization-sensitive optical coherence tomography of human burn scars: birefringence quantification and correspondence with histologically determined collagen density
}

Mariëlle E. H. Jaspers

Fabio Feroldi

Marcel Vlig

Johannes F. de Boer

Paul P. M. van Zuijlen 


\title{
In vivo polarization-sensitive optical coherence tomography of human burn scars: birefringence quantification and correspondence with histologically determined collagen density
}

\author{
Mariëlle E. H. Jaspers, ${ }^{a, b, c, d, \dagger}$ Fabio Feroldi, ${ }^{e, t, \dagger}$ Marcel Vlig, ${ }^{b}$ Johannes F. de Boer, ${ }^{e}$ and \\ Paul P. M. van Zuijlen ${ }^{a, b, c, d}$ \\ ${ }^{a}$ Red Cross Hospital, Burn Center, Beverwijk, The Netherlands \\ ${ }^{b}$ Association of Dutch Burn Centers, Beverwijk, The Netherlands \\ ${ }^{c}$ Red Cross Hospital, Department of Plastic, Reconstructive and Hand Surgery, Beverwijk, The Netherlands \\ dVU University Medical Center, Department of Plastic, Reconstructive and Hand Surgery, Amsterdam Movement Sciences, Amsterdam, \\ The Netherlands \\ ${ }^{e}$ VU University, LaserLaB Amsterdam, Department of Physics and Astronomy, Amsterdam, The Netherlands
}

\begin{abstract}
Obtaining adequate information on scar characteristics is important for monitoring their evolution and the effectiveness of clinical treatment. The aberrant type of collagen in scars may give rise to specific birefringent properties, which can be determined using polarization-sensitive optical coherence tomography (PS-OCT). The aim of this pilot study was to evaluate a method to quantify the birefringence of the scanned volume and correlate it with the collagen density as measured from histological slides. Five human burn scars were measured in vivo using a handheld probe and custom-made PS-OCT system. The local retardation caused by the tissue birefringence was extracted using the Jones formalism. To compare the samples, histograms of birefringence values of each volume were produced. After imaging, punch biopsies were harvested from the scar area of interest and sent in for histological evaluation using Herovici polychrome staining. Two-dimensional en face maps showed higher birefringence in scars compared to healthy skin. The Pearson's correlation coefficient for the collagen density as measured by histology versus the measured birefringence was calculated at $r=0.80(p=0.105)$. In conclusion, the custom-made PS-OCT system was capable of in vivo imaging and quantifying the birefringence of human burn scars, and a nonsignificant correlation between PS-OCT birefringence and histological collagen density was found. $\odot$ The Authors. Published by SPIE under a Creative Commons Attribution 3.0 Unported License. Distribution or reproduction of this work in whole or in part requires full attribution of the original publication, including its DOI. [DOI: 10.1117/1.JBO.22.12.121712]
\end{abstract}

Keywords: optical coherence tomography; scar; collagen; birefringence; scar assessment.

Paper 170537SSRR received Aug. 14, 2017; accepted for publication Dec. 4, 2017; published online Dec. $20,2017$.

\section{Introduction}

Obtaining adequate information on scar characteristics is important for monitoring their evolution and necessary to evaluate the effectiveness of clinical treatments. ${ }^{1,2}$ Most of the scar characteristics that can be distinguished (e.g., thickness, relief, pliability and surface area/contraction) are largely affected by the aberrant type of collagen that is present in scars. ${ }^{3-5}$ Accordingly, treatment modalities are considered to influence the scar's morphometry (i.e., collagen), and therefore, it would be desirable to have a noninvasive imaging device, providing absolute structural information on collagen.

Optical coherence tomography (OCT) is a low coherence interferometric technique, whose potential for high resolution imaging (1 to $20 \mu \mathrm{m}$ ) of biological tissue at depths of up to $2 \mathrm{~mm}$ has been demonstrated in the last two decades. ${ }^{6,7}$ With polarization-sensitive OCT (PS-OCT), the polarimetric properties of a sample can be determined. For example, when light travels through a birefringent sample, its polarization state changes. ${ }^{8}$ By quantifying this change, the magnitude of the

\footnotetext{
*Address all correspondence to: Fabio Feroldi, E-mail: f.feroldi@vu.nl

†These authors contributed equally.
}

birefringence can be assessed, which can be used as a parameter for the differentiation of tissue types. This technique has been used to study the morphology of normal and diseased skin. ${ }^{9-11}$ Furthermore, wound healing has been studied noninvasively by PS-OCT, and in burns, preclinical studies were performed to detect collagen denaturation in the dermal skin layer, which is a potential optical marker due to thermally induced conformational changes. ${ }^{12,13}$ Thereafter, PS-OCT was applied in vivo to two pediatric burn patients and showed the vasculature and birefringence as parameters for characterizing burn wounds. ${ }^{14}$

In burn scars, the large quantity of unidirectional aligned collagen fibers in the dermis ${ }^{15}$ may give rise to strong birefringence, which seems therefore a suitable structure to characterize with a PS-OCT system, as done by Gong et al. ${ }^{16}$ In their clinical study, a commercial PS-OCT system equipped with a custommade scanner was used to quantify the birefringence of scarred tissue, in vivo. Despite the accurate removal of the tissue vasculature, the single-incident polarization state nature of the system poses the challenge that it could become aligned with the tissue optic axis, impairing the determination of the birefringence. Moreover, they used the slope of the cumulated phase retardation to produce two-dimensional (2-D) en face maps of the tissue birefringence, which requires no trivial operations to account for possible phase wrapping. Other studies 
from the same group have exploited different kinds of non-PSOCT contrast to assess burn wounds and scars, such as attenuation coefficient ${ }^{17}$ and quantification of the diameter and density of the tissue vasculature. ${ }^{18}$

The purpose of this pilot study was to extend these previous results by comparing PS-OCT results with histological collagen assessment. To do so, a handheld probe and custom-made PS-OCT system was used, measuring human burn scars in vivo. We developed a method to determine the overall volume birefringence, reducing the large amount of data to a single number, allowing to compare it with collagen density, as measured by histological analysis.

\section{Material and Methods}

\subsection{Study Design and Patients}

An experimental pilot study was performed at the Department of Plastic, Reconstructive and Hand Surgery of the Red Cross Hospital in Beverwijk, the Netherlands. In order to be eligible to participate in this study, a subject must meet all of the following criteria: age $\geq 18$ years, suffering from a (hypertrophic) burn scar, and scheduled for excision of the scar. The exclusion criteria were a language barrier and/or pregnancy. The latter was determined by a pregnancy test in all women prior to scanning. The regional medical ethics committee approved the study protocol with registration number M014-033 (NL46182.094.14). In total, five patients were included and all patients gave written informed consent.

\subsection{Optical Coherence Tomography Imaging System}

In order to provide robust imaging in the clinical setting, a single-mode fiber based PS-OCT setup was used (Fig. 1). The system has been extensively explained in a previous publication from our group. ${ }^{19}$ In brief, the system consists of a $1310 \mathrm{~nm}$ swept source laser (Axsun Technologies Inc., Billerica, Massachusetts), an optical trigger, balanced detectors (PDB430C, Thorlabs Inc., Newton, New Jersey), and a high-speed digitizer (ATS9350, Alazar Inc., Pointe-Claire, Canada). The interferometer features a polarization delay unit to depth-multiplex two orthogonal polarization states, a circulator in the sample arm to maximize sensitivity, and a fiber-based polarization-diversity detection module.

The handheld device is composed of a collimator, two galvo scanning mirrors, and an objective (LSM03, Thorlabs Inc.) to focus the light on the skin, all enclosed in a POM-C plastic housing and window of N-BK7 glass (Fig. 2). A sterile plastic cover (equipment cover 17587, Microtek Medical B.V., Zutphen, the Netherlands) covered the whole module when operating the device on the patient. The galvo mirrors were driven by analog signals delivered by a home-built controller, synchronized with the OCT acquisition trigger signal. The scanning region ranged $3 \mathrm{~mm} \times 3 \mathrm{~mm}$, consisting of 250 cross-sectional images (in the $x$ - $z$ plane), each comprising 2000 A-lines sampled in $\mathrm{k}$-space with 1024 points. The phase retardation between orthogonal polarization states caused by the tissue birefringence was extracted from the data following the Jones matrix formalism. ${ }^{20}$ Coherent signal composition was used to enhance the signal-to-noise ratio (SNR) and contrast of both the intensity and phase retardation images. ${ }^{19,21}$

\subsection{Optical Coherence Tomography Processing}

We chose to extract local retardation values, because they represent the morphology of the tissue birefringence and simplify its quantification. ${ }^{11}$ We based our algorithm on an implementation extensively described by Makita et al. ${ }^{22}$ First, we matched in depth the detected electric fields for the orthogonally polarized depth encoded incident fields, yielding a total of four complex signals (two per incident polarization state) per spatial location. The global phase of each spatial location was calculated from the complex sum of its four field components and consequently subtracted from each field. This operation aligned the components in complex space, allowing efficient spatial averaging of the individual fields by a Gaussian-shaped averaging kernel with a size of twice the system resolution ( $z$-direction: $32 \mu \mathrm{m}$ with full width at half maximum (FWHM) $16 \mu \mathrm{m}, x$-direction: $40 \mu \mathrm{m}$ with FWHM $20 \mu \mathrm{m}$ ).

The local phase retardation was determined by comparing the measured complex electric fields resolved at a certain depth $z$ with the ones measured at a depth $z+\Delta z$, with $\Delta z$ corresponding to a distance of $49.3 \mu \mathrm{m}$, assuming a tissue refractive index of 1.43 .

By assuming that the optical fibers of the system are pure retarders and that the tissue diattenuation is negligible, one

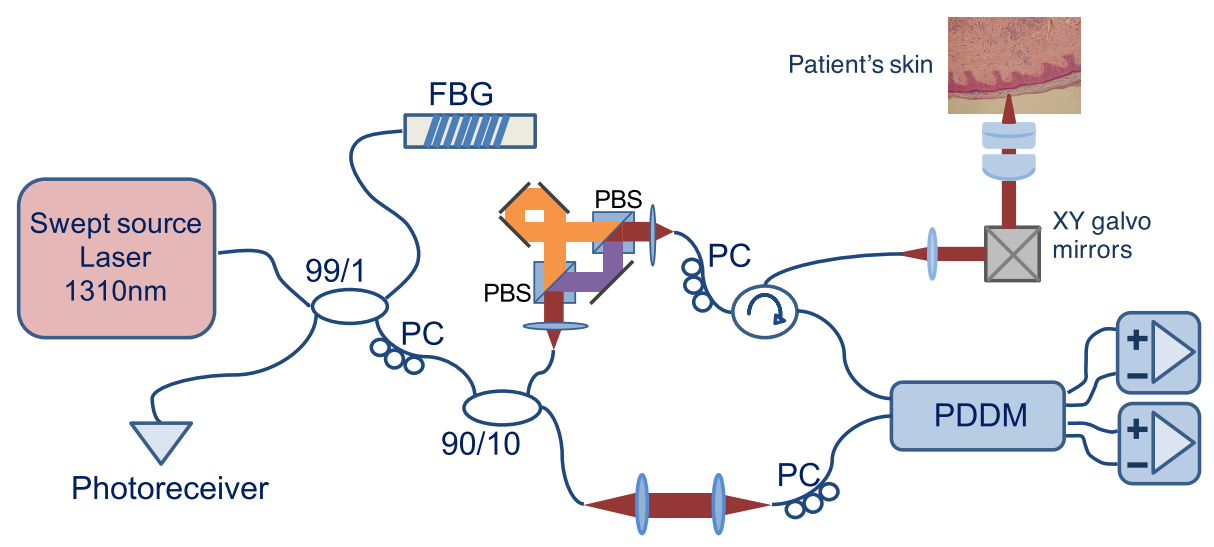

Fig. 1 FBG, fiber Bragg grating; PC, polarization controller; PBS, polarization beam splitter; C, circulator; PDDM, polarization diversity detection module; and $99 / 1$ and $90 / 10$, fiber-optic couplers with their respective coupling ratio. 

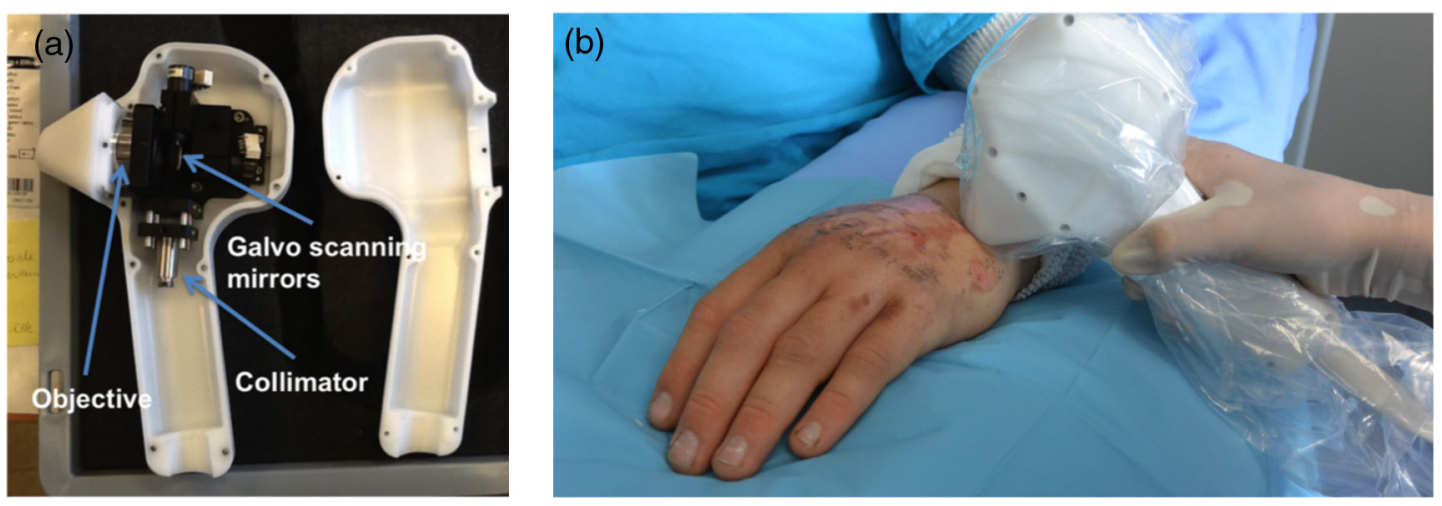

Fig. 2 (a) Handheld probe components. When in function, an optical fiber was connected to the collimator and-together with the electronic cables for driving the galvo mirrors-inserted in an umbilical cord. (b) Typical clinical use of the handheld probe. The sterile plastic sheet covered the whole device.

can eliminate the influence of the system itself on the measurement. ${ }^{8}$ The equation that describes the transmission of the electric field in the sample eventually becomes: ${ }^{8,22}$

$$
\mathbf{E}_{\text {out }}(z) \mathbf{E}_{\text {out }}^{-1}(z+\Delta z)=\mathbf{J}_{\text {out }}(z) \mathbf{J}_{S}(z, \Delta z) \mathbf{J}_{\text {out }}(z)^{-1},
$$

where the bold letters indicate complex matrices. $\mathbf{E}_{\text {out }}(z)$ and $\mathbf{E}_{\text {out }}(z+\Delta z)$ are the Jones vectors measured at depth $z$ and $z+\Delta z$, respectively. $\mathbf{J}_{\text {out }}(z)$ represents the Jones matrix that models the OCT system as a pure retarder from depth $z$ of the sample to the detectors. $\mathbf{J}_{S}(z, \Delta z)$ represents the Jones matrix of the sample between the locations $z$ and $z+\Delta z$.

Due to the property of similar matrices, the left side of Eq. (1) shares the same eigenvalues of the inner matrix of the right side. By determining the absolute phase difference of the eigenvalues, one can extract the phase difference cumulated by the OCT signal between the locations $z$ and $z+\Delta z$. This operation was repeated for every location with an $\mathrm{SNR}>5 \mathrm{~dB}$, providing a cross-sectional map of the round-trip local retardation of the tissue. Finally, we denoised these maps by applying a twicethe-resolution sized Gaussian-shaped averaging kernel.

To provide an absolute measurement of birefringence, we then converted the local phase retardation values into birefringence $(\Delta n)$, using

$$
\Delta n=n \times \Delta \varphi \times \lambda_{0} / 4 \pi,
$$

where $n$ is the refractive index of the material, $\Delta \varphi$ is the roundtrip local phase retardation, and $\lambda_{0}$ is the central wavelength of the OCT system.

In order to summarize the data in more intuitive 2-D maps for clinical evaluation, we calculated the median birefringence of each A-line and plotted it in a 2-D en face image [see Figs. 4(b), 4(c), 5(c), and 5(d)].

In order to reach our goal of providing a birefringence assessment of the whole scanned tissue volume, we determined all the birefringence values in the volume and processed them in a single histogram [see Figs. 4(d) and 5(e)]. We retained only the values with a birefringence higher than $0.18 \times 10^{-3}$ because lower values were associated with situations of poor contact between the tissue and the plastic cover, which still had a high SNR because of the scattering induced in the ultrasonic gel. The median birefringence was used as a single quantitative number characterizing the total tissue volume birefringence. We finally compared this metric with the collagen density as determined by histological analysis.

\subsection{Measurement Procedure}

Two researchers (M.J. and F.F.) performed the OCT measurements on the same day as the scheduled excision of the scar. Prior to imaging, the scar area of interest was marked using a permanent marker. The probe was covered with the sterile plastic bag, sterile gel was applied on the patient's scar, and subsequently, the probe was gently placed onto the scar. The acquisition time of an OCT scan was $\sim 60 \mathrm{~s} / \mathrm{scar}$. Hereafter, the patient's adjacent healthy skin-serving as a reference standard-was imaged. In total, we acquired 10 OCT scans: 5 burn scars and 5 adjacent controls. After imaging, a punch biopsy of $3 \mathrm{~mm}$ in diameter within the scar area of interest was obtained. This sample was fixed in $4 \%$ paraformaldehyde and sent in for histological evaluation. In addition to the scar biopsies, five healthy skin samples obtained from the Euro Tissue Bank Beverwijk were measured, as it was found unethical to obtain skin biopsies from the patients' healthy skin. These samples served as healthy biopsy controls. The healthy skin samples were related to the anatomical location of the imaged scars.

\subsection{Histological Evaluation}

After dehydration, samples were embedded in paraffin, cut perpendicular to the skin surface on a microtome $(5 \mu \mathrm{m} \mathrm{sec}-$ tions), and mounted on glass slides. Of every sample, histology slices were obtained from the middle part of the biopsy and used for Herovici polychrome staining to differentiate between newly formed and mature collagen. ${ }^{23,24}$ Images were taken using a digital camera (Nikon DS-Ri2, Nikon, Amsterdam, the Netherlands) mounted on an Axioskop40FL microscope (Zeiss, Badhoevedorp, the Netherlands). Using digital image analysis software (NIS-Elements 4.4, Nikon), we were able to select only mature collagen and discard other structures (Fig. 3). Using the Herovici stain, young collagen will color blue, whereas mature collagen will stain red [Fig. 3(a)]. To determine the mature collagen density, the image was converted to a ratio view based on the red-green-blue color channels. The ratio between the red and blue channel was visualized [Fig. 3(b)], after which a threshold was set to select only the mature collagen. This was obtained by selecting the pixels with a red/blue ratio greater than 2. The threshold was overlaid on the original image in order to 
(a)

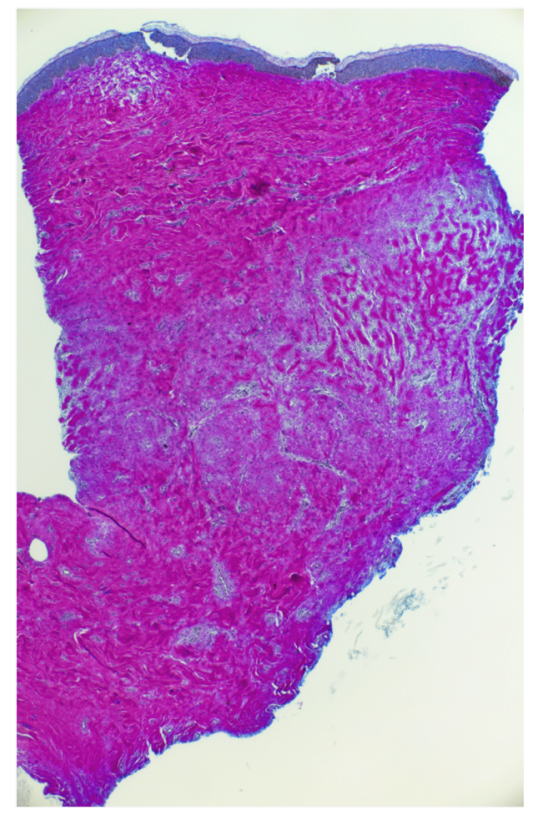

(b)

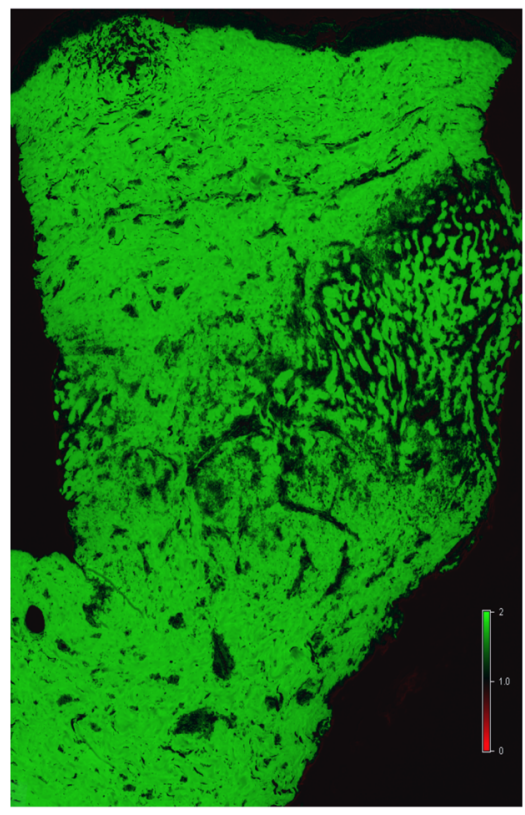

(c)

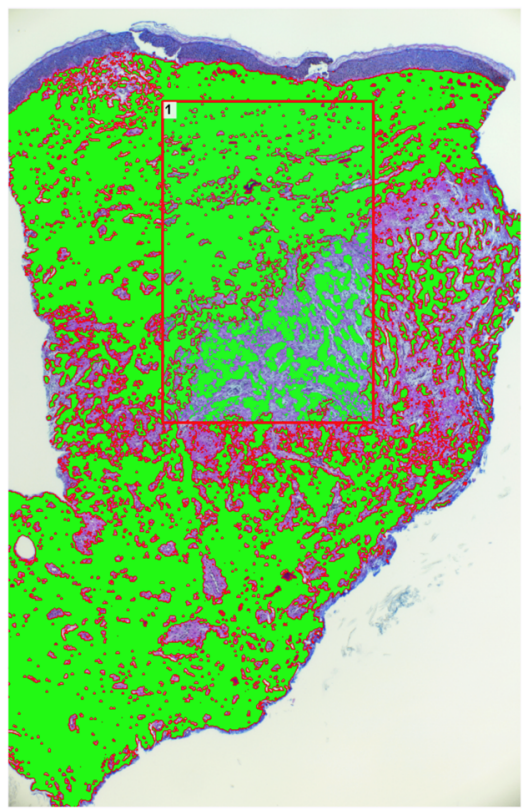

Fig. 3 Example of (a) Herovici polychrome staining and (b) and (c) digital image analysis of scar case $\mathrm{S}-01$. The digital image analysis software only selects mature collagen (green) and discards other structures (black in the panel (b) and purple in the panel (c).

Table 1 Patient and scar characteristics, collagen density percentages, and PS-OCT birefringence values.

\begin{tabular}{|c|c|c|c|c|c|c|}
\hline Case/gender & $\begin{array}{c}\text { Age of patient } \\
\text { (years) }\end{array}$ & $\begin{array}{l}\text { Scar age } \\
\text { (months) }\end{array}$ & Scar location & $\begin{array}{l}\text { Collagen density } \\
\text { scars (\%) }\end{array}$ & $\begin{array}{l}\text { Birefringence scars, } \\
\text { median } \Delta n\left(10^{-3}\right)\end{array}$ & $\begin{array}{l}\text { Birefringence control, } \\
\text { median } \Delta n\left(10^{-3}\right)\end{array}$ \\
\hline S-01/F & 26 & 30 & Sternum & 67.8 & 1.55 & 1.11 \\
\hline S-02/F & 52 & 6 & Axilla & 7.8 & 0.70 & 1.14 \\
\hline S-03/F & 52 & 29 & Arm & 44.7 & 1.21 & 1.03 \\
\hline S-04/F & 25 & 7 & Neck & 43.7 & 1.45 & 1.37 \\
\hline S-05/M & 34 & 24 & Chin & 71.3 & 1.21 & 1.27 \\
\hline
\end{tabular}

Note: The birefringence $\Delta n$ is dimensionless.

visualize the final result [Fig. 3(c)]. The collagen density analysis took place in a predefined square of $1500 \times 500 \mu \mathrm{m}^{2}$, located at the top of the sample. The collagen density of the scars was expressed as the percentage of red surface within the predefined square.

\section{Results}

The general characteristics of all subjects included in this study are presented in Table 1 . The median scar age was 24 months, ranging from 6 to 30 months postburn. The collagen density of the scars, as determined by histological evaluation using Herovici staining, is also shown in Table 1, as well as the scars' birefringence as assessed by PS-OCT. The mean collagen density in the scars was $47.1 \%$, compared to $32.9 \%$ in the five healthy skin samples supplied by the Euro Tissue Bank.

The OCT results of a 7-month-old hypertrophic burn scar in a 25-year-old female patient (S-04) are shown in Fig. 4. The scar was caused by a wood fire burn reaching up to the neck and face. Figures 4(b) and 4(c) show the 2-D en face maps for the scar and the adjacent control region, the latter indicated by the dotted circle. These maps show a distinction between the high birefringence within the scar (yellow) and low birefringence in healthy skin (blue), accompanied with some confined variation. The median scar birefringence in this case was 1.45 compared to 1.37 for the control region. The scar's collagen density obtained by histological evaluation was $43.7 \%$.

Figure 5 shows a 24-month-old hypertrophic burn scar of a 34-year-old Caucasian male patient (S-05). The scar developed after severe facial burn injury caused by a gas explosion in 2013. PS-OCT scans were performed in the outlined circular regions indicated in Figs. 5(a) and 5(b). Figures 5(c) and 5(d) depict the 2-D en face maps for, respectively, the scar and control region located in the neck. The birefringence is noticeably higher in the scar (yellow) than in healthy skin (cyan and blue). The probability histogram in Fig. 5(e) shows the same pattern as in case S-04, a high peak at low birefringence values, and slightly higher probability at high birefringence values. The median scar birefringence was 1.21 compared to 1.27 for the control region 

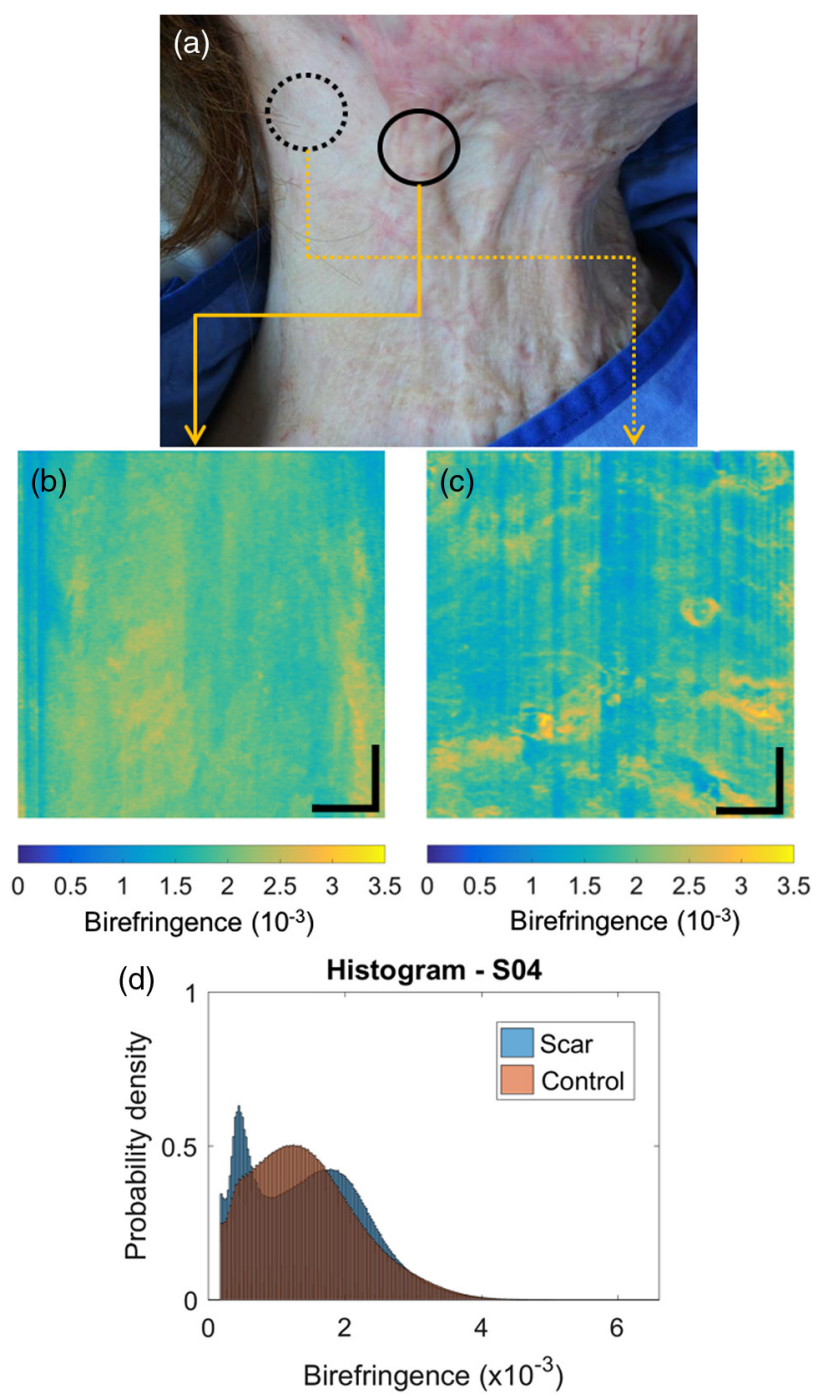

Fig. 4 Birefringence and en face maps of included burn scar (S-04) and adjacent healthy skin. (a) Photograph of area of interest. (b) and (c) En face birefringence maps of, respectively, scar and healthy skin. Scale bars are $500 \mu \mathrm{m}$. (d) Probability histogram of the birefringence of the whole volume for scar and healthy skin (i.e., control).

in the neck. In this case, the collagen density as determined by histological evaluation was $71.3 \%$.

Figure 6 shows the collagen density as measured by histology in relation to the birefringence values as measured by PSOCT. The Pearson's correlation coefficient was calculated at $r=0.80$ with a $p$-value of 0.105 and a sample size of 5 . The $p$-value is sample size dependent and can decrease significantly with an increased sample size of similar observations. We expect that a larger sample size will increase the significance of the correlation.

\section{Discussion}

In this study, we demonstrated the use of a custom-made PS-OCT system for the assessment of human burn scars in vivo in a clinical setting. Scar and healthy skin birefringence was quantified, and after scar excision, biopsies were sent in for histological evaluation to correlate collagen density with PSOCT established birefringence.
In this pilot study, we focused on depiction of the dermis in particular (covering $1.5 \mathrm{~mm}$ in depth beginning at the skin surface), since it is hypothesized that most of the birefringence (i.e., the rate at which the polarization state of light changes) is caused by the amount of dermal collagen formed during the proliferative phase of wound healing. On propagation through the epidermis, which is only 50 to $150 \mu \mathrm{m}$, the polarization state shows little change and a lack of birefringence.

When considering the 2-D en face maps, nearly all scars showed higher birefringence (yellow) than adjacent healthy skin, however, the quantified birefringence values in scars were varying. In case $\mathrm{S}-02$, we observed a lower birefringence in the scar compared to the control area. A possible explanation may be the age of this scar, which was 6 months, thereby reflecting a relatively immature scar. When looking at detail, the histological section of case S-02 shows granulocytes or inflammatory cells in the predefined region interest, whereas the collagen bundles are situated deeper in the biopsy sample (Fig. 7). As a result, both the collagen density percentage and birefringence value were low. Hence, it may be suggested that birefringence as obtained with PS-OCT is more or less a reflection of scar formation.

Over the years, several measurement tools and scar scales have been developed to assess hypertrophic scars. ${ }^{5,25,26}$ In this way, scar evolution can be monitored and the efficacy of various treatment strategies could be evaluated. However, these tools or scar scales only define the thickness/height or pliability of the scar, thereby providing an indirect measure of the amount of dermal collagen. On the other hand, several detailed methods are available, thereby quantifying collagen morphometry in terms of geometrical features, such as bundle thickness and spacing, but this includes invasive histological evaluations. ${ }^{27}$ PS-OCT could potentially be used to monitor scar collagen and collagen maturation in a detailed and noninvasive manner. Furthermore, previous research also suggests conventional OCT to be useful to assess acute wound healing in human skin by obtaining the mean gray scale value (MGV; i.e., intensity level). ${ }^{7,28}$ In these studies, the MGV was significantly increased 6 weeks after wounding and OCT assessed architectural changes corresponded with the different phases of wound healing (i.e., inflammatory, proliferative, and remodeling phase) as evaluated histologically, suggesting that conventional OCT can assess the amount of fibrosis and monitor wound healing noninvasively.

It is challenging to exactly align the OCT images with the histological slides. Although the position of the OCT probe can be marked on the patient, thereby ensuring that the direction of the B-scans in the $x-z$ plane can be traced back, it is rather difficult to mark the small biopsy sample and to cut $5 \mu \mathrm{m} \mathrm{sec-}$ tions in the identical direction. Moreover, as the OCT probe was held by hand, slight movements must have occurred during imaging, as well as small unintentional motions due to respiration and pulsation. Solutions have been previously proposed, such as fixing the scan head to the patient, ${ }^{29}$ yet, this may introduce additional patient discomfort. Furthermore, as the collagen density varies among different anatomical areas, ${ }^{30}$ it seems best to interpret birefringence values in relation to healthy skin, rather than absolute birefringence scar values. In our study, we measured adjacent skin by PS-OCT, in order to minimize the influence of variation. Maybe the exact contralateral area would even be more suitable, but in patients with extensive burn scars, it is frequently challenging to find a control area, as most of the body surface area can be affected by scarring. Therefore, we aimed to 

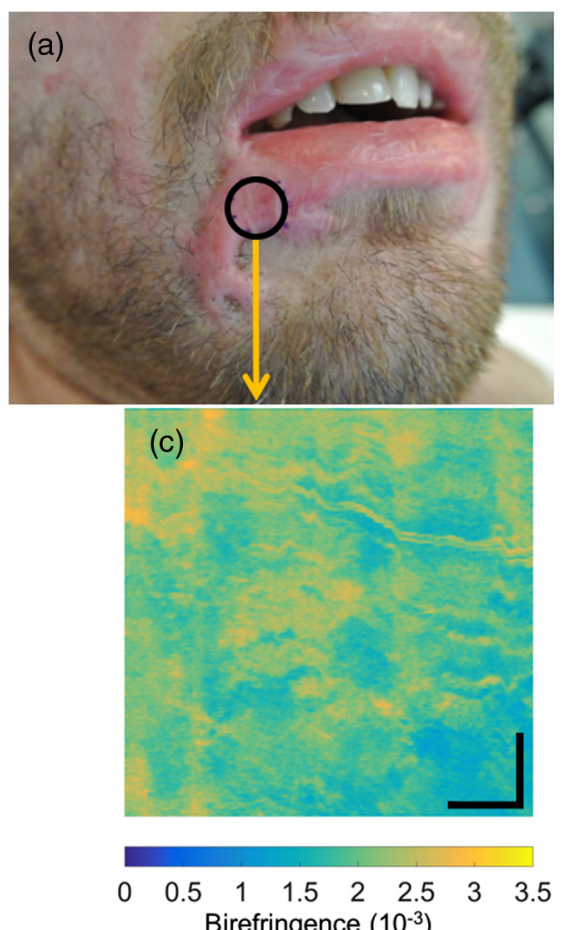
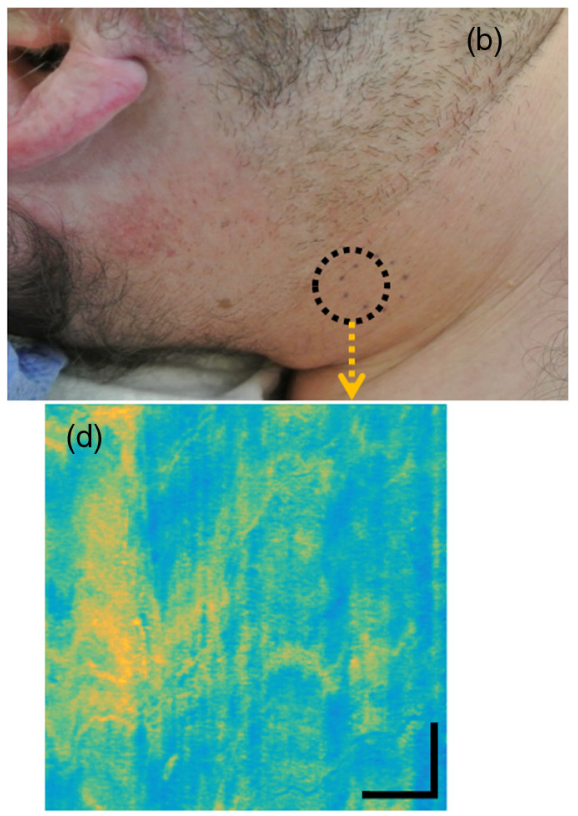

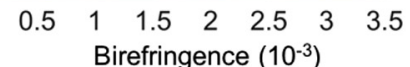

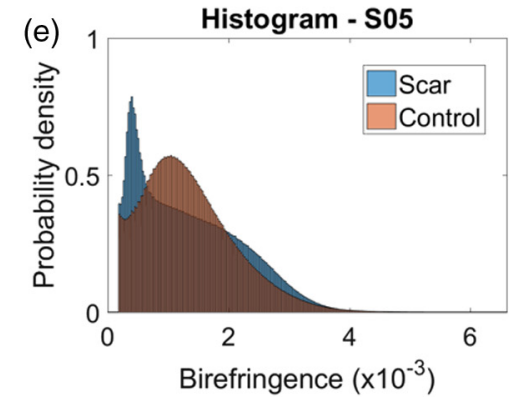

Fig. 5 Birefringence and en face maps of included hypertrophic burn scar (S-05) and adjacent healthy skin. (a) and (b) Photographs of areas of interest. (c) and (d) En face birefringence maps of, respectively, scar and healthy skin. Scale bars are $500 \mu \mathrm{m}$. (e) Probability histogram of the birefringence of the whole volume for scar and healthy skin (i.e., control).

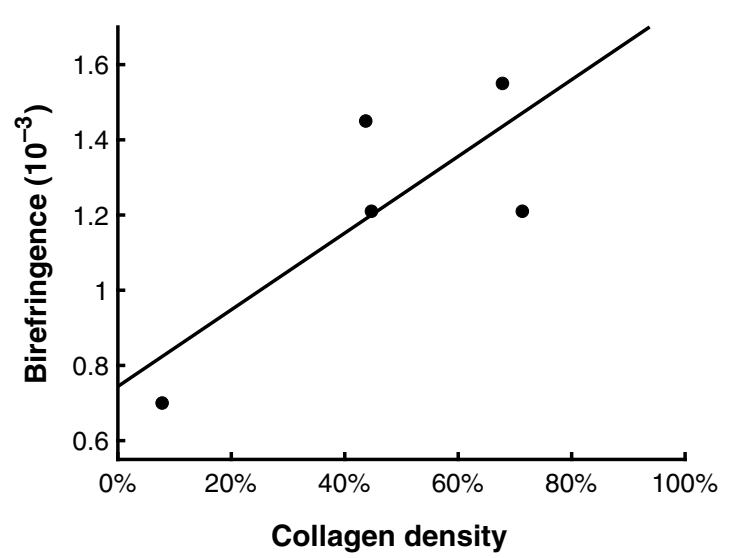

Fig. 6 Scatterplot of collagen density percentages determined by histological evaluation against birefringence values as measured with PS-OCT. select an adjacent control area that reflected the anatomical place of the scar best. Last, the imaging depth of OCT is limited to the most superficial $1.5 \mathrm{~mm}$ of the scar while the scar biopsies showed a dermal thickness of 3 to $4 \mathrm{~mm}$. It is acknowledged that the PS-OCT calculations are therefore not covering the entire scar. Nevertheless, as earlier emphasized, the superficial dermis appears to provide valuable information on the scar's maturity.

In conclusion, we presented a method to quantify scar birefringence of the scanned volume and correlate it with the collagen density as measured from histological slides. An association was found between the birefringence values and collagen density $(p=0.105)$ for a sample size of 5 . We expect the correlation to become statistically significant with an increase in the number of sampled subjects. Future work has to be performed to be able to study human scar development over time, thereby requiring multiple measurements and thus less time for data processing and interpretation of each measurement. Based on this pilot study, PS-OCT could be a useful tool to apply in future research or clinical practice, offering a new parameter containing information on scar collagen density. 
(a)

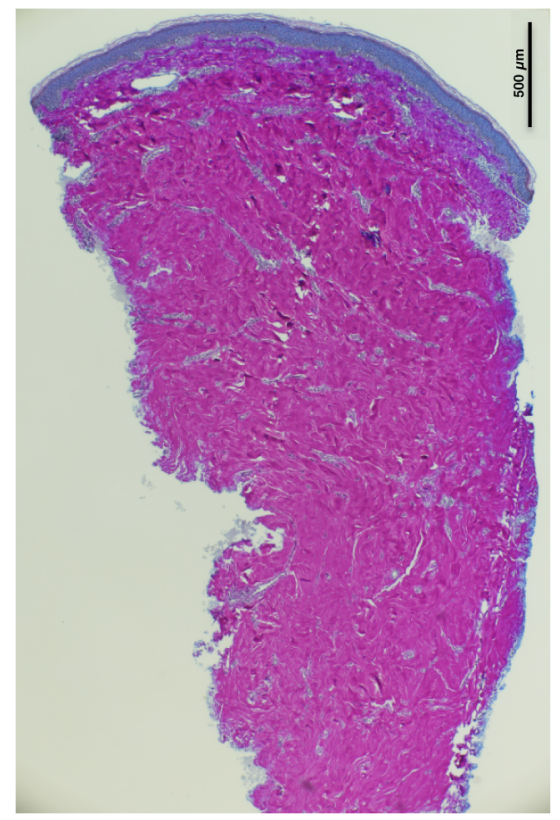

(b)

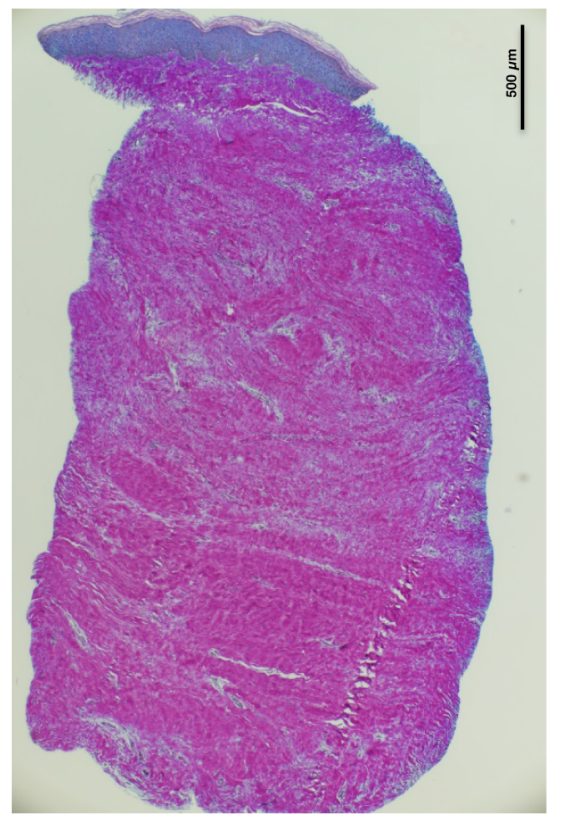

(c)

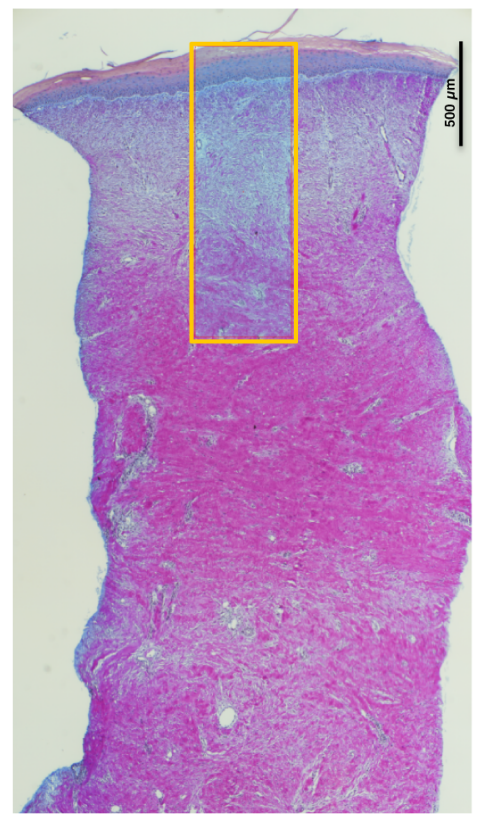

Fig. 7 Histological slides after Herovici staining of case (a) S-05, (b) S04, and (c) S-02. Note that in case S-02, the predefined region of interest (yellow rectangle of $1500 \times 500 \mu \mathrm{m}$ ) is almost entirely covered by granulocytes or inflammatory cells (light purple). This may be an explanation for the low percentage $(7.8 \%)$ of collagen density found in this case. Scale bar: $500 \mu \mathrm{m}$.

\section{Disclosures}

None of the authors has a financial interest in any of the products, devices, or drugs mentioned in this manuscript.

\section{Acknowledgments}

The authors thank J. J. A. Weda for his assistance in designing and developing the OCT scanner, and B. Braaf and M. Graefe for useful discussion on the PS-OCT processing. We thank Thijs Bink for helping in acquiring the data. We also thank all patients for participating and collaborating in this study.

\section{References}

1. T. A. Mustoe et al., "International clinical recommendations on scar management," Plast. Reconstr. Surg. 110(2), 560-571 (2002).

2. G. G. Gauglitz et al., "Hypertrophic scarring and keloids: pathomechanisms and current and emerging treatment strategies," Mol. Med. 17(1-2), 113-125 (2011)

3. P. D. Verhaegen et al., "Objective scar assessment tools: a clinimetric appraisal," Plast. Reconstr. Surg. 127(4), 1561-1570 (2011).

4. L. J. Draaijers et al., "Skin elasticity meter or subjective evaluation in scars: a reliability assessment," Burns 30(2), 109-114 (2004).

5. M. B. van der Wal et al., "A clinimetric overview of scar assessment scales," J. Burn Care Res. 33(2), e79-e87 (2012).

6. S. Yun et al., "High-speed optical frequency-domain imaging," Opt. Express 11(22), 2953-2963 (2003).

7. N. S. Greaves et al., "Optical coherence tomography: a reliable alternative to invasive histological assessment of acute wound healing in human skin?" Br. J. Dermatol. 170(4), 840-850 (2014).

8. B. H. Park et al., "Jones matrix analysis for a polarization-sensitive optical coherence tomography system using fiber-optic components," Opt. Lett. 29(21), 2512-2514 (2004).

9. J. Welzel et al., "Optical coherence tomography of the human skin," J. Am. Acad. Dermatol. 37(6), 958-963 (1997).

10. S. Sakai et al., "In vivo evaluation of human skin anisotropy by polarization-sensitive optical coherence tomography," Biomed. Opt. Express 2(9), 2623-2631 (2011).
11. W. C. Y. Lo et al., "Longitudinal, 3D imaging of collagen remodeling in murine hypertrophic scars in vivo using polarization-sensitive optical frequency domain imaging," J. Invest. Dermatol. 136(1), 84-92 (2016).

12. S. M. Srinivas et al., "Determination of burn depth by polarization-sensitive optical coherence tomography," J. Biomed. Opt. 9(1), 207 (2004).

13. B. H. Park et al., "In vivo burn depth determination by high-speed fiberbased polarization sensitive optical coherence tomography," J. Biomed. Opt. 6(4), 474 (2001).

14. K. H. Kim et al., "In vivo imaging of human burn injuries with polarization-sensitive optical coherence tomography," J. Biomed. Opt. 17(6), 066012 (2012).

15. P. D. Verhaegen et al., "Differences in collagen architecture between keloid, hypertrophic scar, normotrophic scar, and normal skin: an objective histopathological analysis," Wound Repair Regener. 17(5), 649-656 (2009).

16. P. J. Gong et al., "Imaging of skin birefringence for human scar assessment using polarization-sensitive optical coherence tomography aided by vascular masking," J. Biomed. Opt. 19(12), 126014 (2014).

17. P. Gong et al., "Assessment of human burn scars with optical coherence tomography by imaging the attenuation coefficient of tissue after vascular masking," J. Biomed. Opt. 19(2), 021111 (2014).

18. Y. M. Liew et al., "In vivo assessment of human burn scars through automated quantification of vascularity using optical coherence tomography," J. Biomed. Opt. 18(6), 061213 (2013).

19. J. Li et al., "Polarization sensitive optical frequency domain imaging system for endobronchial imaging," Opt. Express 23(3), 3390-3402 (2015).

20. B. H. Park and J. de Boer, Optical Coherence Tomography: Technology and Applications, W. Drexler and J. G. Fujimoto, Eds., Chapter 22, Springer, New York (2008).

21. J. Li and J. F. de Boer, "Coherent signal composition and global phase determination in signal multiplexed polarization sensitive optical coherence tomography," Opt. Express 22(18), 21382-21392 (2014).

22. S. Makita, M. Yamanari, and Y. Yasuno, "Generalized Jones matrix optical coherence tomography: performance and local birefringence imaging," Opt. Express 18(2), 854-876 (2010).

23. C. Herovici, "[Picropolychrome: histological staining technic intended for the study of normal and pathological connective tissue]," Rev. Fr. Etud. Clin. Biol. 8, 88-89 (1963). 
24. P. P. Anthony, "Manual of histological demonstration techniques," J. Clin. Pathol. 28(4), 339-339 (1975).

25. N. Brusselaers et al., "Burn scar assessment: a systematic review of objective scar assessment tools," Burns 36(8), 1157-1164 (2010).

26. Z. Tyack et al., "A systematic review of the quality of burn scar rating scales for clinical and research use," Burns 38(1), 6-18 (2012).

27. P. D. Verhaegen et al., "Collagen bundle morphometry in skin and scar tissue: a novel distance mapping method provides superior measurements compared to Fourier analysis," J. Microsc. 245(1), 82-89 (2012).

28. N. S. Greaves et al., "Skin substitute-assisted repair shows reduced dermal fibrosis in acute human wounds validated simultaneously by histology and optical coherence tomography," Wound Repair Regener. 23(4), 483-494 (2015).

29. K. Konig et al., "Clinical optical coherence tomography combined with multiphoton tomography of patients with skin diseases," J. Biophotonics 2(6-7), 389-397 (2009).

30. M. C. Pierce et al., "Birefringence measurements in human skin using polarization-sensitive optical coherence tomography," J. Biomed. Opt. 9(2), 287 (2004).

Biographies for the other authors are not available. 